\title{
Co-expression of methyl parathion hydrolase TCP degrading genes in genetically engineered bacterium
}

\author{
Liu Zhiyuan ${ }^{1, a}$ \\ ${ }^{1}$ Department of environmental engineering,Shenyang Institute of Technology,Fushun \\ 113122, China \\ a54105222@163.com
}

\begin{abstract}
Keywords: organophosphorus; biodegradation; TCP; chlorpyrifos
Abstract. Organophosphorus compounds are the most widely used insecticides, accounting for an estimated $38 \%$ of total pesticides used globally. Chlorpyrifos is known for its significant use in agriculture; although chlorpyrifos is defined as a moderately toxic substance, its residues create detrimental effects to the environment. The intermediate metabolite of chlorpyrifos, 3,5,6-trichloropyridinol (TCP), is strongly resistant to microbial degradation, making its toxicity to the environment much more significant than chlorpyrifos itself. Unfortunately, the environmental and toxicological problems of TCP is not internationally and domestically consistent. It is agriculturally important to determine the degradation pathway of chlorpyrifos, detect its microbial metabolites, and subsequently find efficient methods to repair chlorpyrifos contaminated soil. In order to degrade chlorpyrifos and its intermediate metabolite TCP simultaneously, the methyl parathion hydrolase gene $(m p d)$ and the TCP degrading gene $(t c p 3 \mathrm{~A})$ were connected to plasmid $p E T$-Duet to generate plasmid $p E T D u e t^{T M}-m p d-t c p 3 \mathrm{~A}$. The plasmid $p E T D u e t^{T M}-m p d-t c p 3 \mathrm{~A}$ was then inserted into E.coli BL21 (DE3) to construct a genetically engineered strain, capable of the co-expression of methyl parathion hydrolase and TCP hydrolase. This study provides an efficient strategy to biodegrade chlorpyrifos and TCP by the construction of a genetically engineered strain of E. coli. Using the initial enzyme to degrade chlorpyrifos and TCP , the degradation rate can reach $68.8 \%$ and $47.9 \%$ in 100 minutes, respectively.
\end{abstract}

\section{Introduction}

Unlike other organophosphate pesticides, the intermediate metabolites of chlorpyrifos exhibit strong inhibition to microbial degradation. Since the first experimental use of chlorpyrifos in 1965, few chlorpyrifos and TCP degrading strains have been reported. The first reported chlorpyrifos degrading strains, Flavobacterium sp.[1] and Pseduomonas diminuta [2],were derived from diazinon and parathion contaminated soil. Chlorpyrifos was degraded in liquid by a co-metabolism pathway, but could not be used as the sole carbon source by degrading bacterial strains. TCP has antibiotic capabilities, causing the chlorpyrifos degrading stains to be inhibited in soil. In alkaline soils, chlorpyrifos was converted to 3,5,6-trichloropyridinol by a co-metabolism pathway. In order to rupture the TCP pyridine ring, chlorine ions must be removed; the biodegradation of chlorpyrifos was inhibited by the toxic inhibition of microorganisms by chlorpyrifos and TCP [3]. Most previous studies have simply confirmed that chlorpyrifos is converted to TCP by the degrading bacteria, but TCP was not mineralized further in the environment $[4,5,6]$. Although this conversion removed the toxicity of chlorpyrifos in the environment, it produced the toxic compound 3,5,6-trichloropyridinol (TCP), which cannot be utilized by microbes themselves [7, 8]. Because TCP strongly inhibits microbial communities, as the chlorpyrifos degraded, a secondary pollution of the environment occurred. The current study provides significant progress in the study of widely used pesticides, and their harm to the ecological environment due to intermediate metabolites produced in the degradation of chlorpyrifos, which has recently attracted widespread attention worldwide [9].

Microorganisms degrade a large variety of pesticides, and are thought to be a significant future development in solving pesticide pollution. Chlorpyrifos, one of the most widely used organophosphorus insecticides, is difficult to degrade in the environment. Few microorgannisms which are capable of degrading chlorpyrifos have been isolated; some of the rare examples are 
Micrococcus sp[10]., Enterobacter sp.[11], Stenotrophomonas sp.[12] and Sphingomonas sp.[13]. However, TCP cannot be degraded by these bacteria. Previously, only two bacteria shown to degrade TCP have been isolated (Pseudomonas sp. and Alcaligenes faecalis), both of which which can degrade TCP completely under controlled laboratory conditions. Previous research on Pseudomonas sp. demonstrates that the TCP degradation mechanism is a synergistic effect of ultraviolet and microbial degradation, through a reductive dechlorination pathway.

Current research indicates that chlorpyrifos degradation was extremely slow under natural conditions, and the restrictive factor for chlorpyrifos degradation by microorganisms is the inhibition of microorganism growth by TCP. Therefore, it was crucial to screen strains which could degrade both chlorpyrifos and its hydrolysis product TCP.

In this study, a chlorpyrifos degradating strain Pseudomonas YF-5, has been isolated from pesticide polluted soil in preliminary experiments. The coding gene (mpd) for methyl parathion hydrolase, which can degrade chlorpyrifos, was cloned in Pseudomonas YF-5. In order to simultaneously degrade chlorpyrifos and its intermediate metabolite, TCP, the methyl parathion hydrolase gene (mpd) and the TCP degrading gene (tcp3A) were incorporated into plasmid pET-Duet to generate plasmid pETDuetTM-mpd-tcp3A. The plasmid pETDuetTM-mpd-tcp3A was then inserted into E.coli BL21 (DE3) to construct a genetically engineered strain capable of co-expression of methyl parathion hydrolase and TCP hydrolase. This study provides an efficient strategy to biodegrade chlorpyrifos and TCP by the construction of a genetically engineered bacterial strain.

\section{Materials and methods}

Bacterial strains and plasmids

E.coli BL21(DE3) and E.coli DH5 $\alpha$ (Tiangen) were used throughout the experiement as the expression host and cloning host, respectively. Plasmid $p E T D u e t^{T M}$ (Tiangen) was used for the coexpression of the $m p d$ gene and the tcp3A gene. Plasmid T-mpd carrying the mpd gene and T-tcp $3 A$ carrying the tcp3A gene (GeneBank: GQ451605) [14] were used as the PCR templates. Additional reagents rTaq DNA polymerase, T4 DNA ligas, HindIII, Bam H I, Xho I, and Nde I were obtained from TaKaRa Biotechnology (Dalian) Co, Ltd.

\section{Media and culture conditions}

Chlorpyrifos ( $>98 \%$ purity) and 3,5,6-ttrichloro-2-pyridinol (TCP, $>95 \%$ purity) were purchased from Jiangsu Hongjing Chemical Limited Company, Jiangsu, China. Methyl parathion was obtained from Shenyang Research Institute of Chemical Industry, Liaoning, China. All other reagents used in this research were of analytical grade.

\section{Construction of the expression plasmids}

PCR was utilized to amplify the $m p d$ and $t c p 3 A$ genes in order to construct an $m p d$ and a $t c p 3 A$ co-expression plasmid. The upstream and downstream oligonucleotide primers of the $m p d$ gene were obtained: $P_{\text {mpd-up }}$ : 5'-GAATTCATATGCCCCTGAAGAAC-3' and P $_{m p d \text {-down }}$ : 5'-GAATTCTCGAG CTTGGGGTTGACGA CCG-3', and the HindIII restriction site and BamH I restriction site (underlined) were inserted into these primers, respectively. Using plasmid pMD18-T-mpd as the PCR template to amplify the mpd gene, the PCR fragment was cloned into the HindIII - BamH I restriction sites of $p E T D u e t^{T M}$ to produce recombinant plasmid $p E T D u e t^{T M}-m p d$.

The upstream and downstream primers of $t c p 3 A$ gene were obtained: $\mathrm{P}_{t c p 3 A}$-up: 5'-GAATTCATA TGCCCCTGAAGAAC-3, and P $\mathrm{P}_{t c p 3 A}$-down: 5'-GAATTCTCGAGCTTGGGGTTGACG CG-3', respectively. A Nde I restriction site was inserted before the tcp $3 A$ start codon, in addition to a Xho $I$ restriction site at the stop codon (underlined). The PCR fragment was cloned into the Nde $I-$ Xho I restriction sites of $p E T D u e t^{T M}-m p d$ to produce recombinant plasmid $p E T D u e t^{T M}{ }_{-m p d-t c p 3 \mathrm{~A}}$. Simultaneously, the cloned PCR fragment was inserted into the Nde $I-X h o I$ restriction sites of 
the $p E T D u e t^{T M}$ to build a recombinant plasmid $p E T D u e t^{T M}-t c p 3 \mathrm{~A}$ as a control. The construction scheme is shown in Figure1. Three recombinant plasmids were designed to generate a His-tag and S-tag sequences for detection and purification of target protein. The correct sequence of the insert was confirmed by gene sequencing.

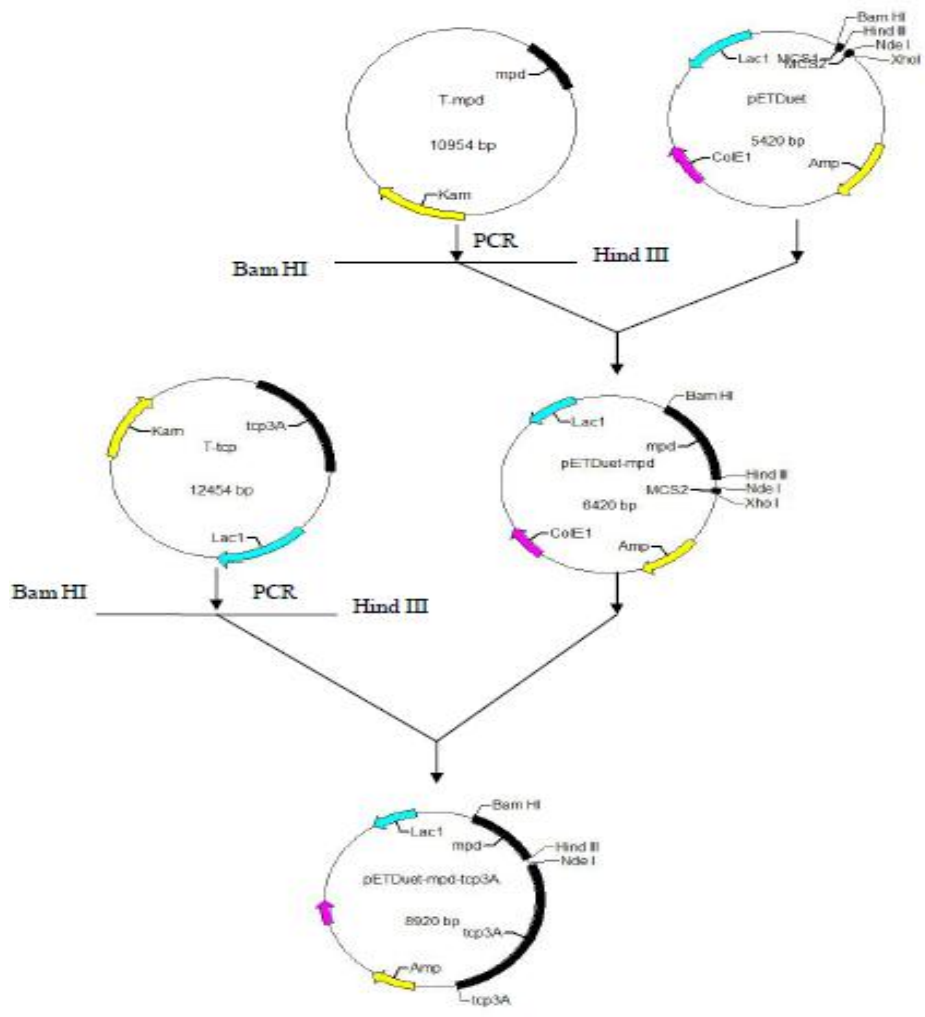

Fig 1. Construction of recombinant plasmid $p E T D u e t^{T M}-m p d-t c p 3 A$.

Transformation and expression of E.coli BL-21(DE3)

Expression research was conducted using E.coli BL-21(DE3). Transformation was performed according to the $\mathrm{pET}$ System Manual( $10^{\text {th }}$ edn. TB055, Novagen2002). The plasmids $p$ ETDuet $^{T M}{ }_{-m p d,}$ EETDuet ${ }^{T M}$-tcp3A, and $p E T D u e t^{T M}{ }_{-m p d-t c p 3 \mathrm{~A}}$ were successfully transformed. One white colony was removed with a sterile toothpick from the LB Amp medium (LA) plates containing antibiotic, and inoculated into LA medium $\left(200 \mathrm{rpm}, 37^{\circ} \mathrm{C}\right.$ shake culture, 12 hours $)$. PCR technology was utilized to choose the positive recombinant plasmid which included the target gene; The strans harboring the recomibinant plasmid were then induced by $1 \mathrm{mM}$ of IPTG for 7 hours in LB medium containing $50 \mu \mathrm{g} \mathrm{mL} \mathrm{m}^{-1}$ kanamycin at $37^{\circ} \mathrm{C}$. The expression products were determined by $10 \%$ sodium sulfate-polyacrylamide gel electrophoresis (SDS-PAGE).

\section{Enzyme extract}

The E.coli BL-21(DE3) harboring the recomibinant plasmid $p E T D u e t^{T M}-m p d-t c p 3 \mathrm{~A}$ was induced by $1 \mathrm{mM}$ of IPTG for 7 hours in LB medium containing $50 \mu \mathrm{g} \mathrm{mL}-1$ kanamycin at $37^{\circ} \mathrm{C}$, and the bacteria were collected by centrifugation and lysed by ultrasonic.

Quantification of chlorpyrifos and TCP

Chlorpyrifos residue was measured by high-performance liquid chromatography according to the National Standard GB/T14552-2003 "Measurement of organic phosphorus pesticides in water and soil by Chromatography." 
The quantitative analysis was performed using gas chromatography HP 5890. A fused silica capillary column was employed $(30 \mathrm{~mm} \times 0.25 \mathrm{~mm} \times 0.25 \mu \mathrm{m}$, Shenyang Institute of Applied Ecology, Chinese Academy of Sciences, Shenyang, China). The temperatures of the injection port and the detector were set to $240 \mathrm{C}$ and $300 \mathrm{C}$, respectively. The oven temperature was increased linearly from $150 \mathrm{C}$ to $260 \mathrm{C}$ at a rate of $5 \mathrm{C} \mathrm{min}^{-1}$. The detection limit of gas chromatography for chlorpyrifos was $0.001 \mathrm{mg} \mathrm{L}^{-1}$. A volume of $10 \mu \mathrm{L}$ sample solution was injected into the injection port.

\section{Result and discussion}

Cloning the mpd gene and functional expression in E. coli

According to the GenBank registered $m p d$ gene sequence (No. AF338729), $\mathrm{P}_{\text {mpd-up }}$ and $\mathrm{P}_{\text {mpd-down }} \mathrm{PCR}$ primers were used to amplify the full-length $m p d$ gene, using the total DNA of pMD18-T- $m p d$ as the template. Fig. 2 demonstrates the result of agarose gel electrophoresis; the amplified band was approximately $996 \mathrm{bp}$ in length. Subsequently, a comparison of experimental gene sequences with the gene sequences in GenBank demonstrates $99 \%$ similarity in the nucleic acid sequence of the $m p d$ gene cloned from Stenotrophomonas sp. YC-1 [15] GenBank sequences (registration number: DQ677027) demonstrate 99\% similarity with the $m p d$ gene at the nucleic acid sequence cloned from the diphtheria mp-4 bacterial strain (GenBank registration No. AY627036). The gene sequence has been submitted to the GenBank database, registration number KF584916.

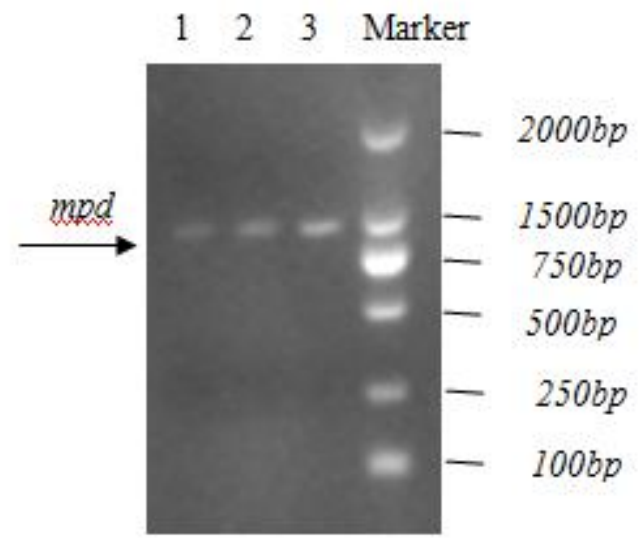

Fig. 2 PCR amplification of $m p d$ gene. Lane 1,2, and 3 demonstrate the purity of the mpd gene; lane 4 represents the gene marker.

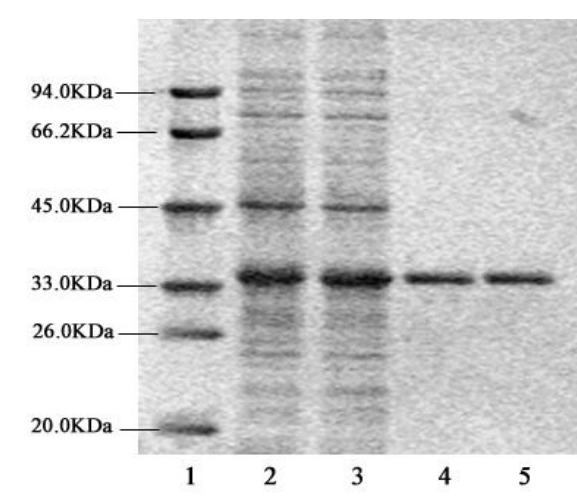

Fig.3 Expression of $m p d$ gene in E.coli BL21(DE3). Lane 1: protein marker; lanes 2 and 3: E. coli cell lysis after IPTG induction; lanes 4 and 5: purified MPH.

The $m p d$ gene (after the deletion of the first 35 amino acids of the N-terminal) was cloned into the expression vector $p E T D u e t^{T M}$, to construct the recombinant expression vector $p E T D u e t^{T M}-m p d$. The plasmid $p E T D u e t^{T M}$-mpd was digested using HindIII + Bam H I .The result was verified with $1 \%$ agarose gel electrophoresis. One $996 \mathrm{bp}$ band and one linear $p E T D u e t^{T M}$ vector appeared after double digestion, indicating the successful construction of the $p E T D u e t^{T M}-m p d$.

As shown in Fig. 3, pETDuet ${ }^{T M}$-mpd was successfully introduced into the gene sequence of E.coli BL21(DE3), adding 1mM IPTG, induced 8 hours. Cells were disrupted by ultrasonic waves and then analyzed by SDS-polyacrylamide gel electrophoresis to determine total protein. These results indicate that the mpd gene in recombinant E.coli BL21(DE3) achieved a high level of expression.

tcp3A gene cloning and functional expression in E. coli

According to the GenBank registered tcp3A gene sequence (GenBank accession no. GQ451605), Primers $\mathrm{P}_{\text {tcp3A-up }}$ and $\mathrm{P}_{t c p 3 A \text {-down }}$ were used to amplify the full length of the $t c p 3 A$ gene, using pMD18-T-tcp3A carrying the $t c p 3 A$ gene as the template. Agarose gel electrophoresis results, shown in Figure 4, demonstrate that the amplified band was a $2.5 \mathrm{~kb}$ amplified fragment. 


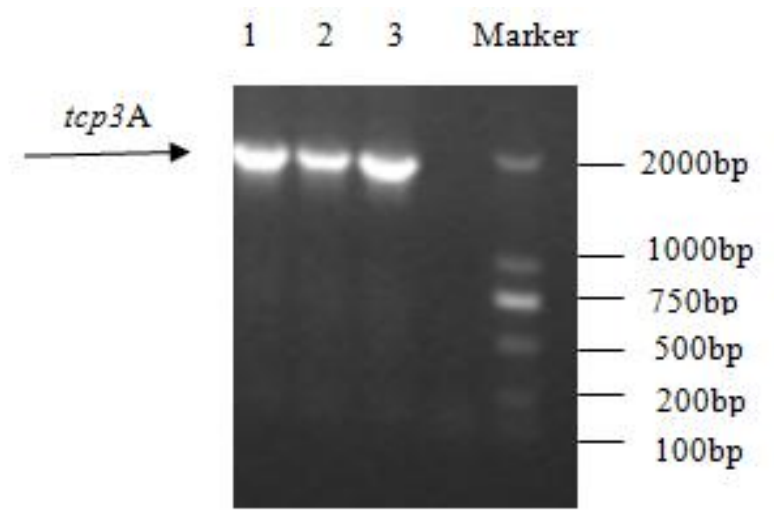

Fig. 4 PCR amplification of $t c p 3 A$ gene. Lanes 1,2,3: purity of $t c p 3 A$ gene; lane 4: gene marker.

The $t c p 3 A$ gene (after the deletion of the first 35 amino acids of the $\mathrm{N}$-terminal) was cloned into the expression vector $p E T D u e t^{T M}$, to construct the recombinant expression vector $p E T D u e t^{T M}$-tcp $3 A$. The resultant plasmid was digested using Nde $I$ and $X h o I$ and verified with $1 \%$ agarose gel electrophoresis. A $2.5 \mathrm{~kb}$ band and a linear pETDuet vector appeared after double digestion, indicating the successful construction of vector $p E T D u e t^{T M}-t c p 3 A$. As shown in Fig. 5, $p E T D u e t^{T M}$-tcp3A was inserted into E.coli BL21(DE3). After the induction by $1 \mathrm{mM} \mathrm{IPTG}$ for 8 hours, cells were disrupted by ultrasonic waves and then analyzed by SDS-polyacrylamide gel electrophoresis to determine their total protein. These results indicate that the recombinant TCP achieved a high level of expression in E.coli BL21(DE3).

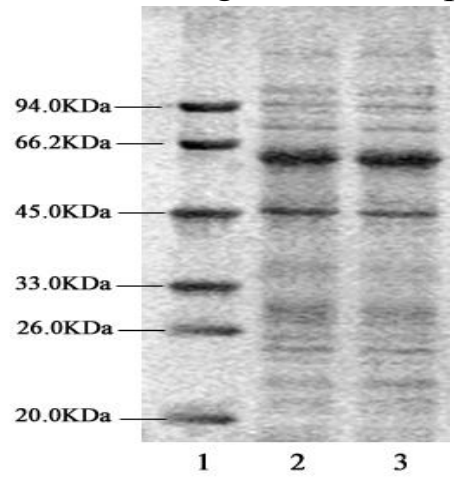

Fig. 5 Expression of tcp3A gene in E.coli BL21(DE3). Lane 1: protein marker; lanes 2 and 3: E. coli cell lysis after IPTG induction.

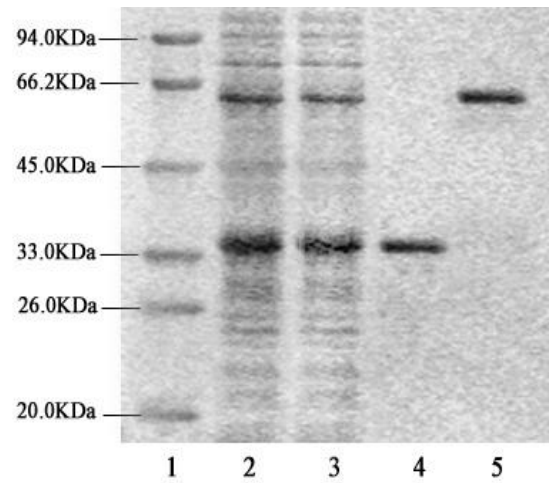

Fig. 6 Expression of $m p d$ and $t c p 3 A$ genes in E.coli BL21(DE3). Lane 1: protein marker; lane 2 and 3, E. coli cell lysis after IPTG induction; lane 4: purified MPH; lane 5: purified TCP degrading enzyme

Construction and expression vector in E.coli BL21(DE3) with tcp3A and mpd gene co-expression

The recombinant plasmid pMD18-T-mpd and $p E T D u e t^{T M}$ was digested by HindIII and BamH I , and the products were connected by T4 DNA Ligase. After DNA purification, positive recombinant plasmid $p E T D u e t^{T M}-m p d$ was obtained The recombinant plasmid $p E T D u e t^{T M}{ }_{-} m p d$ was digested using Nde $I$ and Xho $I$; the tcp3A gene was then inserted into the plasmid $p E T D u e t^{T M}{ }_{-} m p d$ to build recombinant plasmid $p E T D u e t^{T M}-m p d-t c p 3 \mathrm{~A}$. Verification by PCR confirmed that both the $m p d$ gene and the tсp $3 A$ gene fragments were inserted in the recombinant plasmid. Then the plasmid pETDuet ${ }^{T M}{ }_{-m p d-t c p 3 A}$ was transformed into E.coli BL21(DE3).As shown in Fig. 6, the SDS-PAGE results indicate that after the induction of IPTG, the recombinant TCP and MPD achieved a high level of expression in E.coli BL21(DE3). 


\section{Degradion effect of Chlorpyrifos and TCP}

Fig. 7 demonstrates the degrading enzyme changes in a chlorpyrifos and TCP substrate concentration of $100 \mathrm{mg} \mathrm{L}^{-1}$. Results indicate that when $0.3 \mathrm{~mL}$ of the reaction system are added to $3 \mathrm{~mL}$ crude enzyme solution, enzyme degradation efficiency is increased with time to 100 minutes. In 100 minutes, the use of MPH to degrade chlorpyrifos can result in up to $68.8 \%$ degradation, and the use of TCP enzyme to degrade TCP can result in $47.9 \%$ degradation.

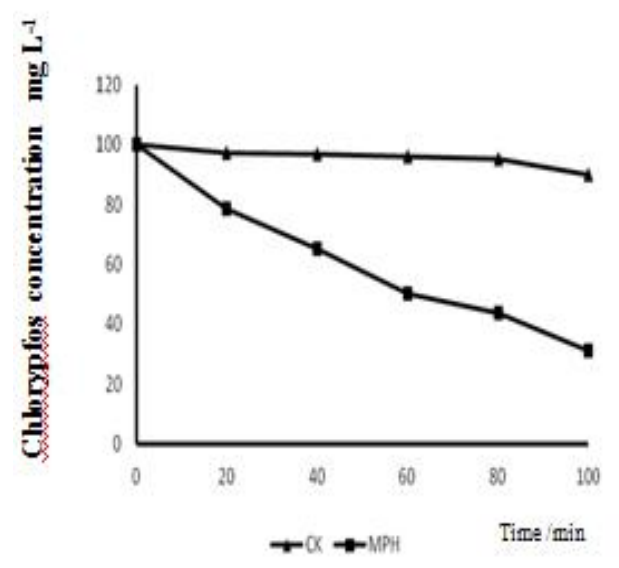

(a) The degradation rate of MPH enzyme.

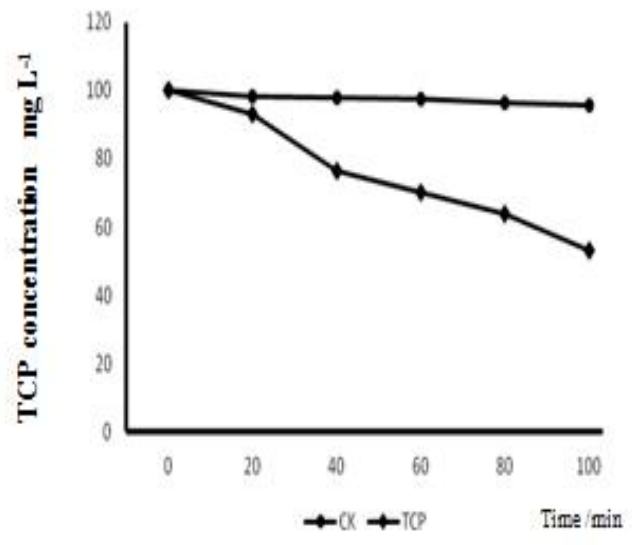

(b) The degradation rate of TCP enzyme.

Fig. 7 The degradation rate of MPH and TCP enzyme in media

\section{Discussion}

1. The $m p d$ gene was cloned from plasmid T-mpd and expressed in E. coli BL21 (DE3). This $m p d$ sequence was submitted to the GenBank database, registration number KF584916.

2. According to previous studies, the $t c p 3 A$ gene was synthesized and cloned into the $p E T D u e t$ plasmid to construct recombinant $p E T D u e t-m p d-t c p 3 A$, which was then inserted into E.coli BL21. The results indicate that $m p d$ and $t c p 3 A$ achieved high expression, with the simultaneous degradation of chlorpyrifos and its intermediate metabolite, TCP.

3. Using the initial enzyme extract to degrade chlorpyrifos and TCP, the degradation rate can reach $68.8 \%$ and $47.9 \%$ in 100 minutes, respectively.

\section{Acknowledgements}

This work was financially supported by the Shenyang Institute of Technology Dr. startup fund (No. BS201405) .

\section{Reference}

[1] N.Sethunathan , T.N Yoshida. Flavobacterium that degrdes diazinon and parathion .Can J Microbiol. Vol.19(1973), p.873-875

[2] C.M Serdar, D.T Gibson, D.M Munnecke, Lancaster JH Plasmid involvement in parathion hydrolysis by Pseudomonas diminuta.Appl Environ Microbiol.Vol.44 (1982), p. 246-249

[3] S.Anwar, F .Liaquat, Q.M. Khan, ZM .Khalid ZM, S. Iqbal. Biodegradation of chlorpyrifos and its hydrolysis product 3,5,6-trichloro-2-pyridinol by Bacillus pumilus strain C2A1. J Hazard Mater vol.168 (2009) p. 400-405 
[4] K.D. Racke,D.A .Laskowski,M.R. Schultz.Resistance of chlorpyrifos to enhanced biodegradation in soil. Journal of Agricultural and Food Chemistry, vol. 38 (1990),p. 1430-1436.

[5] GM .Xu, W. Zheng, YY .Li, SH. Wang, JS. Zhang, et al. Biodegradation of chlorpyrifos and 3,5,6-trichloro-2-pyridinol by a newly isolated Paracoccus sp.TRP. Int Biodeterior Biodegrad Vol. 62(2008),p.51-56.

[6] JQ.Li, J.Liu, WJ.Shen, XL.Zhao, Y.Hou, et al. Isolation and characterization of 3,5,6-trichloro-2-pyridinol-degrading Ralstonia sp. strain T6. Bioresource Technol .Vol. 101 (2010), p.7479-7483.

[7] S.Baskaran, R.Kookana, R.Naidu. Contrasting behaviour of chlorpyrifos and its primary metabolite, TCP (3,5,6-trichloro-2-pyridinol) with depth in soil profiles. Soil. Res.,Vol. 41(2003), p.749-760

[8] D.E.Muscarella, J.F. keown, et al. Evaluation of the genotoxic and embryotoxic potential of chlorpyrifos and its metabolites in vivo and in vitro.Environ mutage, Vol. 6 (1984), p.13-23

[9] I.Horne, Sutherland TD, Harcourt RL, Russell RJ, Oakeshott JG Identification of an opd (organophosphate degradation) gene in an Agrobacterium isolate. Appl Environ Microbiol.,Vol.68(2002),p.3371-3376.

[10] A.Guha, B.Kumari, M.K .Roy.Possible involvement of plasmid in degradation of malathion and chlorpyrifos by Micrococcus sp. Folia Microbiol, Vol.42(1997),p. 574-576

[11] B.K.Singh., A .Walker, J.A.W.Morgan, D.J.Wright. Effect of soil PH on the biodegradation of chlorpyrifos and isolation of a chlorpyrifos-degrading bacterium.Appl Environ Microbiol .Vol. 68(2003),p. 5198-5206

[12] G.M,Xu, W. Zheng, Y.Y Li, S.H Wang, J.S Zhang, et al. Biodegradation of chlorpyrifos and 3,5,6-trichloro-2-pyridinol by a newly isolated Paracoccus sp.TRP. Int Biodeterior Biodegrad .Vol.62(2008),p.51-56.

[13] S.Wen, Ti.K.Lan., Z. Lu, F. Qin.,et al. Genetically modified microorganism Spingomonas paucimobilis UT26 for simultaneously degradation of methyl-parathion and c-hexachlorocyclohexane. Ecotoxicology. Published on line:20 march 2014

[14]K. Renukaradhya., Math. Md .Shah. Isolation of a novel gene encoding a 3,5,6-trichloro-2-pyridinol degrading enzyme from a cow rumen metagenomic library. Biodegradation.Vol.21 (2010), p. 565-573

[15]C.Yang, N. Liu, XM.Guo, CL Qiao. Cloning of mpd gene from a chlorpyrifos-degrading bacterium and use of this strain in bioremediation of contaminated soil. FEMS Microbiol Lett.Vol. 265 (2006) p.118-125. 